\title{
High frequency of enterotoxigenic Bacteroides fragilis and Enterococcus faecalis in the paraffin-embedded tissues of Iranian colorectal cancer patients
}

\author{
Nasibeh Khodaverdi ${ }^{1}$, Habib Zeighami ${ }^{1}$, Ahmad Jalilvand ${ }^{2}$, Fakhri Haghi ${ }^{*}$ and Negar Hesami ${ }^{3}$
}

\begin{abstract}
Background: The association between specific bacteria and colorectal cancer (CRC) has been proposed. Only a few studies have, however, investigated this relationship directly in colorectal tissue with conflicting results. So, we aimed to quantitate Streptococcus gallolyticus, Fusobacterium spp, Enterococcus faecalis and enterotoxigenic Bacteroides fragilis (ETBF) in formalin-fixed and paraffin-embedded (FFPE) colorectal tissue samples of Iranian CRC patients and healthy controls.
\end{abstract}

Methods: A total of 80 FFPE colorectal tissue samples of CRC patients $(n=40)$ and healthy controls $(n=40)$ were investigated for the presence and copy number of above bacterial species using quantitative PCR. Relative quantification was determined using $\triangle \triangle C T$ method and expressed as relative fold difference compared to reference gene.

Results: Relative abundance and copy number of E. faecalis and ETBF were significantly higher in CRC samples compared to control group. E. faecalis was more prevalent than ETBF in tumor samples. Frequency of ETBF and E. faecalis in late stages (III/IV) of cancer was significantly higher than early stages (I/II). We did not detect a significant difference in abundance of $S$. gallolyticus and Fusobacterium spp between two groups.

Conclusion: Our study revealed the higher concentration of E. faecalis and ETBF in FFPE samples of CRC patients than controls. However, additional investigations on fecal and fresh colorectal cancer tissue samples are required to substantiate this correlation.

Keywords: Bacteroides fragilis, Colorectal cancer, Enterococcus faecalis, Fusobacterium spp, Streptococcus gallolyticus

\section{Background}

Colorectal cancer (CRC) is one of the most common cancers worldwide, accounting for approximately 1.9 million new cases and 900.000 deaths in 2020 [1, 2]. The etiology of CRC is not fully understood. Several risk factors are associated with initiation and progression of CRC

\footnotetext{
*Correspondence: haghi@zums.ac.ir

1 Department of Microbiology and Virology, School of Medicine, Zanjan University of Medical Sciences, Zanjan, Iran

Full list of author information is available at the end of the article
}

including genetic susceptibility, epigenetic and environmental factors such as diet, obesity, smoking, alcohol consumption and host immunity $[1,3,4]$. Because of high number of false positive cases in CRC screening systems such as Fecal Occult Blood Test (FOBT), identifying the sensitive biomarkers for early detection allows efficient treatment of CRC [1]. Previous studies have demonstrated a causal link between specific bacterial and viral pathogens and cancers such as gastric carcinoma, cervical cancer and hepatocellular carcinoma [3, 5-7]. A possible role of oncogenic bacteria in CRC 
development was first suggested in 1951 when a clinical association between Streptococcus bovis bacteremia/ endocarditis and CRC was reported $[1,5,6]$. Since then, numerous studies have shown the enrichment of fecal or tissue samples of CRC patients with bacterial species such as enterotoxigenic Bacteroides fragilis (ETBF), Fusobacterium nucleatum, Clostridium septicum, Enterococcus faecalis, Streptococcus gallolyticus, Enteropathogenic Escherichia coli and $p k s^{+}$E. coli $[1,4-6]$. Several bacterial-induced carcinogenic mechanisms in CRC have been proposed including Wnt signaling activation, proinflammatory signaling and genotoxicity $[5,6]$. Following bacterial infection and chronic inflammation, changes occur in the cellular microenvironment that leads to precancerous and finally cancerous events [2]. $p k s^{+} E$. coli possesses a genotoxin colibactin, produced by the polyketide synthase genomic island $(p k s)$ that induces DNA damage, cell cycle arrest, mutations and chromosomal instability in eukaryotic cells. The interaction of LPS, FadA (Fusobacterium adhesion protein A) and Fap2 (Fusobacterium autotransporter protein 2) located on the surface of $F$. nucleatum with epithelial cell can affect host immune response via decreased apoptosis, cellular proliferation and DNA repair through activation of the nuclear factor- $\kappa \mathrm{B}(\mathrm{NF}-\kappa \mathrm{B})$ and Wnt signaling pathways $[3,8]$. The possible carcinogenic role of S. gallolyticus in $\mathrm{CRC}$ is mediated by overexpression of cyclooxygenase- 2 (COX2), prevention of apoptosis and promotion of angiogenesis and inflammation. Furthermore, E. faecalis strains produce reactive oxygen species and extracellular superoxide anions that cause DNA damage and chromosomal instability [9]. A significant association between the presence of ETBF in fecal or colon biopsy specimens and CRC has been reported in previous studies. Secreted pro-inflammatory B. fragilis toxin (BFT) cleaves and degrades the extracellular domain of cell surface protein $\mathrm{E}$-cadherin. Loss of E-cadherin triggers $ß$-catenin nuclear signaling, induces $c-m y c$ expression and IL-8 secretion [4, 10]. BFT also causes oxidative DNA damage and stimulates the high expression of IL-17. It is proposed that long-term colonization of colonic epithelial cells with ETBF may increase the risk of CRC $[4,10]$.

Although many studies have showed an association between the colorectal cancer development and intestinal microbiota, not all studies have yielded consistent results. Several studies demonstrated $F$. nucleatum as dominant bacteria in CRC patients, while others suggested B. fragilis as an abundant bacterium associated with CRC [3]. So, more research is needed to find association between specific bacteria and CRC.

Most studies investigating bacterial involvement in cancer development were based on fecal samples, while the composition of intestinal microbiota varies in the fecal and mucosal surface [1, 10]. Formalin-fixed and paraffin embedded (FFPE) archived tissues are extremely valuable sources for molecular diagnostic purposes in colorectal cancer $[1,11]$. So, we used quantitative realtime polymerase chain reaction (qPCR) to investigate the presence and copy number of ETBF, E. faecalis, S. gallolyticus and F. nucleatum in FFPE colon tissue samples of colorectal cancer patients and healthy controls.

\section{Methods \\ Sample collection}

This case-control study was approved by the Research Ethics Committee of Zanjan University of Medical Sciences (IR.ZUMS.REC.1398.481). A total of 80 formalin-fixed, paraffin-embedded (FFPE) colorectal tissue samples from patients diagnosed with colorectal cancer $(n=40)$ and healthy controls $(n=40)$ were collected from archives of Pathology Department of Ayatollah Mousavi hospital in Zanjan Province, Iran. Colorectal tissue samples of CRC patients and control group were collected during colonoscopy. The control group underwent colonoscopy for various reasons and all of them were outpatients. No gastrointestinal disease was reported in control group and normal colon mucosa was confirmed. None of the cases or controls had a pre-colonoscopy chemo- or radiotherapy, previous history of other gastrointestinal diseases and antibiotic therapy within the past 1 month. The clinicopathological parameters including gender, age, family history of cancer, smoking, tumor stage and tumor location were collected according to medical records of patients. Sections of $10 \mu \mathrm{m}$ in thickness from the top of FFPE tissue samples were prepared using a standard microtome with disposable blades and transported to the laboratory of Medical Microbiology. Then, normal and tumor tissues were dissected using sterile scalpels for DNA extraction.

\section{DNA extraction}

DNA extraction from FFPE tissue samples was performed using GeneAll Exgene ${ }^{\mathrm{TM}}$ FFPE Tissue DNA kit (GeneAll Biotechnology, Songpa-gu, Seoul, South Korea). Three sections of $10 \mu \mathrm{m}$ in thickness from FFPE samples was cut up and after deparaffinization, samples were incubated in $180 \mu \mathrm{l}$ lysozyme $(20 \mathrm{mg} / \mathrm{ml})$ for $40 \mathrm{~min}$ at $37^{\circ} \mathrm{C}$. Then, $20 \mu \mathrm{l}$ of proteinase $\mathrm{K}$ solution $(20 \mathrm{mg} / \mathrm{ml})$ was added and incubated at $56^{\circ} \mathrm{C}$ for $1 \mathrm{~h}$. The extraction steps were continued according to the manufacturer's instructions. Also, extraction of DNA from non-tissuecontaining paraffin sections was performed to assess any environmental bacterial contamination of blocks during fixation, embedding and processing.

The concentration and purity of DNA samples were determined using NanoDrop spectrophotometer 
(ND-1000, Nano-Drop Technologies, Wilmington, DE) at 260 and $260 / 280 \mathrm{~nm}$, respectively. DNA samples were stored at $-20^{\circ} \mathrm{C}$ for further analysis.

\section{Quantitative real-time polymerase chain reaction (qPCR)} Oligonucleotide primers targeted to detect conserved sequences specific for ETBF, E. faecalis, S. gallolyticus and Fusobacterium spp were selected from the literature and their specificity was confirmed by Primer BLAST (https://blast.ncbi.nlm.nih.gov/Blast.cgi) (Table 1). A conserved sequence present in all bacteria (universal primer) was also used as internal control in qPCR. First, the specificity of amplification was checked using conventional PCR and the amplicons were analyzed by agarose gel electrophoresis for specific band of amplified products. Then, PCR standardized conditions were used to carry out qPCR. Furthermore, conventional PCR was used to assess contamination of non-tissue-containing paraffin sections by universal primer.

Standard curves were prepared for each run of qPCR using seven 10-fold dilutions of extracted DNA from reference strains of ETBF strain D-134, E. faecalis ATCC 29212, S. gallolyticus ATCC 49147 and F. nucleatum ATCC 25586 and DNA copy number was calculated using the following formula:

Number of copies $=$ Mass (in grams) $\times 6.023 \times 10^{23} /$ Average mol. wt. of a base $\times$ template length. According to the standard curves, samples which did not have a fluorescent signal earlier than the Ct of 35 for S. gallolyticus, Fusobacterium spp and E. faecalis and 37 for ETBF were considered as negative.

Limit of detection (LOD) of primers was also determined using serial dilution of bacterial DNA. This was found to be approximately $10^{2}$ DNA copies for $B$. fragilis and 10 DNA copies for S. gallolyticus, Fusobacterium spp and E. faecalis.

Simplex qPCR was performed in a reaction mixture with total volume of $20 \mu \mathrm{l}$ consisted of $10 \mu \mathrm{l}$ of RealQ
Plus 2x Master Mix Green High ROX ${ }^{\mathrm{TM}}$ (Ampliqon, Denmark), $0.4 \mu \mathrm{M}$ of each the specific primer pairs, $30 \mathrm{ng}$ of DNA in double distilled water. Assays were carried out in duplicate with an Applied Biosystems StepOnePlus ${ }^{\text {TM }}$ Real-Time PCR System. Reported data are the mean values of duplicate qPCR analyses. Amplifications involved an initial denaturation at $95^{\circ} \mathrm{C}$ for $10 \mathrm{~min}$, followed by 40 cycles of denaturation at $95^{\circ} \mathrm{C}$ for $30 \mathrm{~s}$, annealing at $57-60^{\circ} \mathrm{C}$ (Table 1) for $30 \mathrm{~s}$, and extension at $72^{\circ} \mathrm{C}$ for $30 \mathrm{~s}$. Amplification specificity of each run was assessed by melting curve analysis. Relative quantification was also determined using the $2^{-\Delta \mathrm{Ct}}$ method and expressed as relative fold difference compared to the reference gene (16S ribosomal RNA) conserved among all bacteria.

\section{Statistical analysis}

Statistical analysis was performed with the Statistical Package for Social Sciences (SPSS), version 17.0 (SPSS, Inc., Chicago, IL). The data were presented as frequencies for qualitative variables and as means \pm standard error mean (SEM) for quantitative variables. The Mann-Whitney U-test and Fisher's exact tests were used to determine the significance of differences between two groups. $P$ value of $<0.05$ was considered significant.

\section{Results}

The clinicopathological characteristics of patients are shown in Table 2. Briefly, a total of 40 FFPE colon tissue samples from CRC patients (19 males and 21 females) and 40 from healthy control cases ( 22 males and 18 females) were investigated. The mean age of CRC patients was 56.37 years (range 31-86years) and for healthy controls was 60 years (range 20-82). The majority of CRC patients were stage III or IV cancer (65\%), while stages I and II were 10 and $25 \%$, respectively. In CRC patients, $62.5 \%$ of tumors were located in distal colon and $37.5 \%$ were in proximal. The distal colon includes the descending colon

Table 1 Primers used in this study

\begin{tabular}{|c|c|c|c|c|c|}
\hline Bacterial pathogens & Target Gene & Primer sequence $(5-3)$ & Amplicon (bp) & $\begin{array}{l}\text { Annealing } \\
\text { Temperature }\left({ }^{\circ} \mathrm{C}\right)\end{array}$ & Ref \\
\hline Fusobacterium spp & 16 s ribosomal RNA & $\begin{array}{l}\text { CCCAAGCAAACGCGATAAGT } \\
\text { GCGTTGCGTCGAATTAAACC }\end{array}$ & 117 & 58 & {$[1]$} \\
\hline ETBF & $b f t$ & $\begin{array}{l}\text { TGAAGTTAGTGCCCAGATGC } \\
\text { CAGTAAAGCCTTCCAGTCC }\end{array}$ & 150 & 58 & {$[10]$} \\
\hline S. gallolyticus & SodA & $\begin{array}{l}\text { AAGCTGCGACAACTCGCTTT } \\
\text { AAGCGTGTTCCCAAACGTCA }\end{array}$ & 150 & 59 & {$[1]$} \\
\hline E. faecalis & 16 s ribosomal RNA & $\begin{array}{l}\text { CCCTTATTGTTAGTTGCCATCATT } \\
\text { ACTCGTTGTACTTCCCATTGT }\end{array}$ & 144 & 60 & {$[12]$} \\
\hline Universal & 16 s ribosomal RNA & $\begin{array}{l}\text { AAACTCAAAKGAATTGACGG } \\
\text { CTCACRRCACGAGCTGAC }\end{array}$ & 180 & 59 & [13] \\
\hline
\end{tabular}


Table 2 Clinicopathological characteristics of patients

\begin{tabular}{lll}
\hline & CRC $(\boldsymbol{n}=\mathbf{4 0})$ & Control $(\boldsymbol{n}=\mathbf{4 0})$ \\
\hline Male & $19(47.5 \%)$ & $22(55 \%)$ \\
Female & $21(52.5 \%)$ & $18(45 \%)$ \\
Age (Mean \pm SE) & $56.37 \pm 14.7$ & $60 \pm 15.11$ \\
Tumor location & & \\
Distal colon & $25(62.5 \%)$ & - \\
Proximal colon & $15(37.5 \%)$ & - \\
Tumor stages & & \\
I & $4(10 \%)$ & - \\
II & $10(25 \%)$ & - \\
III & $11(27.5 \%)$ & - \\
IV & $15(37.5 \%)$ & $17(42.5 \%)$ \\
Smoking & $15(37.5 \%)$ & $3(7.5 \%)$ \\
Family history & $4(10 \%)$ & \\
\hline
\end{tabular}

and the sigmoid colon and proximal colon includes the cecum, ascending colon and the transverse colon.

$16 \mathrm{~S}$ ribosomal RNA gene was not detected in non-tissue-containing paraffin sections. To determine whether CRC was associated with ETBF, S. gallolyticus, Fusobacterium spp and E. faecalis, we used qPCR to compare the frequency and quantity of the bacteria in colorectal tumors and normal colon tissue. $\log _{10}$ values [means \pm standard deviation (SD)] of DNA copy numbers were calculated for each bacterium. The number of positive samples and the copy numbers of each bacterium in normal and tumor tissues are presented in Table 3. Relative quantification was also determined by the $2^{-\Delta \mathrm{Ct}}$ method and is shown in Table 4. E. faecalis was detected in $85 \%$ of CRC samples and ETBF in $45 \%$ of cases. The presence of E. faecalis and ETBF was higher in tumor samples compared to control group $(P<0.05)$. E. faecalis was more prevalent than ETBF in tumor samples $(P<0.05)$. For ETBF, $\log _{10}$ copies DNA ml ${ }^{-1}$ were 3.8 and 2.7 for CRC samples and control group, respectively $(P=0.003)$. As shown in Table 3, higher concentration of E. faecalis was found in CRC patients than control group $(P \leq 0.05)$. The presence of ETBF and E. faecalis was not comparable when stratifying tumor samples based on cancer stages and cancer location (Tables 5 and 6).

According to results, we did not detect a significant difference in the frequency and DNA copy number of $S$. gallolyticus and Fusobacterium spp between two groups (Tables 3 and 4).

\section{Discussion}

In recent years, several reports suggest that intestinal microbial dysbiosis may be an etiological factor in the initiation and progression of colorectal cancer [1, 2, 14, 15]. Although the enrichment of fecal or tissue samples
Table 3 Presence, copy number and Ct values of bacteria in normal and tumor tissues

CRC $(n=40) \quad$ Control $(n=40) \quad P$ value

Qualitative presenta-
tion, percent patients

positive ${ }^{\text {a }}$

Fusobacterium spp

Yes

No

ETBF

Yes

No

S. gallolyticus

Yes

No

E. faecalis

Yes

No

Range and median

values of $\mathrm{Ct}$

Fusobacterium spp

ETBF

S. gallolyticus

E. faecalis

Log copies DNA $\mathrm{ml}^{-1 \mathbf{b}}$

Fusobacterium spp

ETBF

S. gallolyticus

$\begin{array}{ll}27(67.5 \%) & 24(60 \%) \\ 3(32.5 \%) & 16(40 \%)\end{array}$

$18(45 \%) \quad 6(15 \%)$

$22(55 \%) \quad 34(85 \%)$

$29(72.5 \%) \quad 28(70 \%)$

$11(27.5 \%) \quad 12(30 \%)$

0.48

E. faecalis

$34(85 \%)$

$25(62.5 \%)$

$6(15 \%)$

$15(37.5 \%)$

0.003

a Values noted as number (percentage), Fisher's exact test

${ }^{\mathrm{b}}$ Values noted as log copies DNA ml ${ }^{-1}$, Mann-Whitney test

of CRC patients with bacterial species such as ETBF, $F$. nucleatum, E. faecalis, S. gallolyticus and EPEC has been shown in previous studies, not all reports have consistent results $[1,2,4-6]$. Furthermore, data on CRC-associated microbiota in Iranian populations are scarce. Therefore, in order to find a better understanding of this association, we used qPCR to investigate the presence and copy

Table 4 Quantitation of intestinal bacteria in CRC and controls. Quantitative values are shown as relative difference, calculated by the $2^{-\Delta C t}$ method and expressed as relative fold difference compared to the reference gene. Values shown are mean + SEM

\begin{tabular}{llll}
\hline & CRC $(\mathbf{n}=\mathbf{4 0})$ & Control $(\mathbf{n}=\mathbf{4 0})$ & $P$ value \\
\hline Relative difference & & & \\
Fusobacterium spp & $0.03 \pm 0.04$ & $0.02 \pm 0.03$ & 0.62 \\
ETBF & $0.03 \pm 0.01$ & $0.003 \pm 0.02$ & 0.025 \\
S. gallolyticus & $0.04 \pm 0.01$ & $0.02 \pm 0.02$ & 0.18 \\
E. faecalis & $0.05 \pm 0.03$ & $0.01 \pm 0.02$ & 0.029 \\
\hline
\end{tabular}


Table 5 Presence of bacteria in CRC patients with respect to cancer stage

\begin{tabular}{lllll}
\hline $\begin{array}{l}\text { Stage of cancer } \\
\text { Bacteria }\end{array}$ & $\begin{array}{l}\text { Stage I } \\
(\mathbf{n}=\mathbf{4})\end{array}$ & $\begin{array}{l}\text { Stage II } \\
(\boldsymbol{n}=\mathbf{1 0})\end{array}$ & $\begin{array}{l}\text { Stage III } \\
(\boldsymbol{n}=\mathbf{1 1})\end{array}$ & $\begin{array}{l}\text { Stage IV } \\
(\boldsymbol{n}=\mathbf{1 5})\end{array}$ \\
\hline $\begin{array}{l}\text { Fusobacterium spp } \\
(\boldsymbol{n}=\mathbf{2 7})\end{array}$ & $3(75 \%)$ & $5(50 \%)$ & $8(72.7 \%)$ & $11(73.3 \%)$ \\
$\begin{array}{l}\text { ETBF }(\boldsymbol{n}=\mathbf{1 8}) \\
\text { S. gallolyticus }(\boldsymbol{n}=\mathbf{2 9 )}\end{array}$ & $2(50 \%)$ & $4(40 \%)$ & $5(45.4 \%)$ & $7(46.6 \%)$ \\
E. faecalis $(\boldsymbol{n}=\mathbf{3 4 )}$ & $4(100 \%)$ & $5(50 \%)$ & $11(100 \%)$ & $9(60 \%)$ \\
\hline
\end{tabular}

Table 6 Presence of bacteria in CRC patients with respect to cancer location

\begin{tabular}{lll}
\hline $\begin{array}{l}\text { Tumor location } \\
\text { Bacteria }\end{array}$ & $\begin{array}{l}\text { Proximal colon } \\
(\mathbf{n}=\mathbf{1 5})\end{array}$ & $\begin{array}{l}\text { Distal colon } \\
(\mathbf{n}=\mathbf{2 5})\end{array}$ \\
\hline Fusobacterium spp $(\mathbf{n = 2 7 )}$ & $9(60 \%)$ & $18(72 \%)$ \\
ETBF $(\mathbf{n}=\mathbf{1 8})$ & $7(46.6 \%)$ & $11(44 \%)$ \\
S. gallolyticus $(\mathbf{n}=\mathbf{2 9})$ & $10(66.6 \%)$ & $19(76 \%)$ \\
E. faecalis $(\mathbf{n}=\mathbf{3 4})$ & $12(80 \%)$ & $22(88 \%)$ \\
\hline
\end{tabular}

number of ETBF, E. faecalis, S. gallolyticus and F. nucleatum in FFPE colon tissue samples of Iranian CRC patients and healthy controls. According to our results, relative abundance and copy number of $E$. faecalis and ETBF were significantly higher in CRC samples compared to control group. E. faecalis was more prevalent than ETBF in tumor samples. In our study, we used the $16 \mathrm{~s}$ ribosomal RNA gene as an internal control, but it was better to use a reference gene such as prostaglandin transporter (PGT) for normalization. Several case-control studies have reported that the abundance of ETBF was higher in ulcerative colitis, colorectal adenoma and CRC patients than controls $[3-6,9,10]$. In study conducted by Rezasoltani et al. (2018), higher numbers of ETBF, E. faecalis, F. nucleatum, S. bovis, and Porphyromonas spp. were reported in adenomatous polyp patients in contrast to controls [9]. In our previous study, we demonstrated the association between fecal ETBF and CRC and suggested that fecal detection of ETBF may be a potential biomarker for colorectal cancer diagnosis [4]. Furthermore, Shariati et al. (2021) showed a significantly higher abundance of $B$. fragilis and $F$. nucleatum in fresh frozen biopsies of colorectal lesions of Iranian CRC patients compared to adjacent normal mucosal tissues. However, they could not detect such a relation for S. gallolyticus and EPEC [16].

Enterococcus faecalis as one of the most common Gram-positive cocci in human stools produces extracellular superoxide, hydrogen peroxide and hydroxyl radicals which cause DNA damage in mammalian cells and chromosomal instability that lead to colorectal cancer [17]. We detected higher number of $E$. faecalis in CRC patients compared to control group. In consistent with our results, Balamurugan et al. (2008) demonstrated higher level of $E$. faecalis in stool of CRC patients compared to healthy volunteers. According to their results, population of Eubacterium rectale and Faecalibacterium prausnitzii was decreased approximately fourfold in CRC patients compared to healthy group. These changes in bacterial population in colon could potentially lead to epithelial cell damage and increased turnover and may be an etiological factor of CRC [17]. Furthermore, in study conducted by Zhou et al. (2016), the median abundance of Fusobacterium spp., E. faecalis and ETBF in tumor tissues was significantly higher than adjacent normal tissue and healthy controls [6]. However, in a prospective case cohort study of consecutive colonoscopy patients, fecal E. faecalis population was identified as unstable over $>1$ year and an association between superoxide-producing $E$. faecalis and large colon adenomas or cancer was not found [18].

According to our results, the presence of ETBF and $E$. faecalis was not comparable when stratifying tumor samples based on cancer stages and cancer location.

Despite the noted association with CRC, we did not detect a significant difference in the frequency and copy number of S. gallolyticus and Fusobacterium spp between CRC patients and controls. The association between $S$. gallolyticus endocarditis/bacteremia and CRC is well established [19]. However, none of our patients had a history of bacteremia/bacterial-endocarditis and this may explain the lack of difference in abundance of S. gallolyticus between two groups. In agreement with our results, Mahmoudvand et al. (2017) reported no association between S. gallolyticus and colorectal cancer in paraffinembedded biopsy specimens [20]. Only in studies conducted by Abdulamir et al. (2010) and Farajzadeh Sheikh et al. (2020), S. gallolyticus was detected with higher frequency in CRC patients with and without a history of bacterial endocarditis/bacteremia compared to healthy controls [21, 22]. According to Bundgaard-Nielsen et al. (2019) and Viljoen et al. (2015) studies, S. gallolyticus was not detected in any of colorectal tissue samples. Their findings could potentially be explained through ethnic differences in susceptibility to colorectal colonization of S. gallolyticus or geographical differences in S. gallolyticus distribution. Furthermore, these discrepancies may also be related to application of diverse specimens (like fresh frozen tissues, FFPE and stool) and different detection methods (such as qPCR, pyrosequencing, Fluorescence In Situ Hybridization) used for detection of $S$. gallolyticus $[1,5,16]$. 
In contrast to previous studies [5, 6, 9, 23], we did not observe a difference in abundance of Fusobacterium spp between CRC patients and controls. Furthermore, Bundgaard-Nielsen et al. (2019) showed that F. nucleatum was distributed equally in tumors, paired normal tissue and diverticula, but significantly less present in adenomas [1]. However, F. nucleatum abundance in CRC patients varied between 13 and $87 \%$ in different countries $[5,6,9,16$, 23, 24]. According to Shariati et al. (2021) and Kashani et al. (2020) from Iran, F. nucleatum was highly abundant in CRC tissues (23 and 68\%, respectively) compared to adjacent normal mucosa [16, 24]. Environmental factors such as weight, body mass index, diet, and geographical location may play an active role in bringing about this variation [16].

Our study also has several limitations. One of the limitations was the small number of included samples and the use of formalin fixed colorectal tissue specimens. Since formalin fixation causes cross-linking of DNA-tissue protein, which can prevent amplification. Also, DNA fragmentation may occur in formalin fixed tissue, which may limit our ability to detect bacteria $[1,11,25]$. Because all FFPE samples were handled similarly, we do not expect the formalin fixation to affect the observed differences in bacterial load and prevalence between diagnoses. On the other hand, we had a specific focus on the bacterial species E. faecalis, ETBF, S. gallolyticus and Fusobacterium spp. Further studies are need to investigate a potential role of other bacterial species as E. coli and C. septicum, Prevotella and Acinetobacter in CRC.

\section{Conclusions}

Our study revealed the higher concentration of $E$. faecalis and ETBF in FFPE samples of CRC patients than controls. However, additional investigations on fecal and fresh colorectal cancer tissue samples are required to substantiate this correlation.

\section{Abbreviations \\ CRC: Colorectal cancer; ETBF: Enterotoxigenic Bacteroides fragilis; FFPE: Formalin-fixed and paraffin-embedded; FOBT: Fecal Occult Blood Test; NF-kB: Nuclear factor-kB; COX2: Cyclooxygenase - 2; BFT: B. fragilis toxin.}

\section{Acknowledgements}

The authors are grateful to staff of Pathology department of Ayatollah Mousavi hospital for supporting this study.

\section{Authors' contributions}

$\mathrm{FH}$ and $\mathrm{HZ}$ obtained funding for the study and conceptualized and designed the study. NKH collected and analysis the data. AJ conducted data analysis and $\mathrm{NH}$ interpretation of data and revising the manuscript. All authors have read and approved the final article.

\section{Funding}

This work was supported by Zanjan University of Medical Sciences, Zanjan, Iran (Grant number: A-12-535-25).
Availability of data and materials

All data generated or analyzed during this study are included in the manuscript.

\section{Declarations}

Ethics approval and consent to participate

This study was approved by the Research Ethics Committee of Zanjan University of Medical Sciences (A-12-535-25, IR.ZUMS.REC.1398.481). Authors confirming all the experiment protocol for involving human data was in accordance with the guidelines of institutional or declaration of Helsinki in the manuscript. In this study, archived and verified samples were used, and no new samples were taken from patients. In the previous study, the verbal informed consent was obtained from all study participants and it was approved by the Research Ethics Committee of Zanjan University of Medical Sciences (IR.ZUMS.REC.1398.481).

\section{Consent for publication}

Not applicable.

\section{Competing interests}

The authors declare that they have no competing interest.

\section{Author details}

'Department of Microbiology and Virology, School of Medicine, Zanjan University of Medical Sciences, Zanjan, Iran. ${ }^{2}$ Department of Pathology, School of Medicine, Zanjan University of Medical Sciences, Zanjan, Iran. ${ }^{3}$ Department of Microbiology, Zanjan Branch, Zanjan Islamic Azad University, Zanjan, Iran.

Received: 26 July 2021 Accepted: 14 December 2021

Published online: 22 December 2021

\section{References}

1. Bundgaard-Nielsen C, Baandrup UT, Nielsen LP, Sørensen S. The presence of bacteria varies between colorectal adenocarcinomas, precursor lesions and non-malignant tissue. BMC Cancer. 2019;19(1):399.

2. International Agency for Research on Cancer. Globocan 2020: Cancer fact sheets - colorectal Cancer. IARC.

3. Park CH, Eun CS, Han DS. Intestinal microbiota, chronic inflammation, and colorectal cancer. Intestinal research. 2018;16(3):338-45.

4. Haghi F, Goli E, Mirzaei B, Zeighami H. The association between fecal enterotoxigenic B. fragilis with colorectal cancer. BMC Cancer. 2019;19(1):879.

5. Viljoen KS, Dakshinamurthy A, Goldberg P, Blackburn JM. Quantitative profiling of colorectal cancer-associated bacteria reveals associations between fusobacterium spp., enterotoxigenic Bacteroides fragilis (ETBF) and clinicopathological features of colorectal cancer. PLoS One. 2015;10(3):e0119462.

6. Zhou Y, He H, Xu H, Li Y, Li Z, Du Y, et al. Association of oncogenic bacteria with colorectal cancer in South China. 2016;7:49.

7. Yu LC-H, Wei S-C, NiY-H. Impact of microbiota in colorectal carcinogenesis: lessons from experimental models. Intestinal research. 2018;16(3):346-57.

8. Dahmus JD, Kotler DL, Kastenberg DM, Kistler CA. The gut microbiome and colorectal cancer: a review of bacterial pathogenesis. J Gastrointest Oncol. 2018;9(4):769-77.

9. Rezasoltani S, Asadzadeh Aghdaei H, Dabiri H, Akhavan Sepahi A, Modarressi MH, Nazemalhosseini Mojarad E. The association between fecal microbiota and different types of colorectal polyp as precursors of colorectal cancer. Microb Pathog. 2018;124:244-9.

10. Zamani S, Hesam Shariati S, Zali MR, Asadzadeh Aghdaei H, Sarabi Asiabar A, Bokaie $\mathrm{S}$, et al. Detection of enterotoxigenic Bacteroides fragilis in patients with ulcerative colitis. Gut pathogens. 2017;9:53.

11. Huijsmans CJJ, Damen J, van der Linden JC, Savelkoul PHM, Hermans MHA. Comparative analysis of four methods to extract DNA from paraffin-embedded tissues: effect on downstream molecular applications. BMC Research Notes. 2010;3(1):239. 
12. Cannon K, Byrne B, Happe J, Wu K, Ward L, Chesnel L, et al. Enteric microbiome profiles during a randomized phase 2 clinical trial of surotomycin versus vancomycin for the treatment of Clostridium difficile infection. J Antimicrob Chemother. 2017;72(12):3453-61.

13. Bacchetti De Gregoris T, Aldred N, Clare AS, Burgess JG. Improvement of phylum- and class-specific primers for real-time PCR quantification of bacterial taxa. J Microbiol Methods. 2011;86(3):351-6.

14. Peters BA, Dominianni C, Shapiro JA, Church TR, Wu J, Miller G, et al. The gut microbiota in conventional and serrated precursors of colorectal cancer. Microbiome. 2016;4(1):69.

15. Liu W, Zhang R, Shu R, Yu J, Li H, Long H, et al. Study of the relationship between microbiome and colorectal Cancer susceptibility using 16SrRNA sequencing. Biomed Res Int. 2020;2020:7828392.

16. Shariati A, Razavi S, Ghaznavi-Rad E, Jahanbin B, Akbari A, Norzaee S, et al. Association between colorectal cancer and Fusobacterium nucleatum and Bacteroides fragilis bacteria in Iranian patients: a preliminary study. Infectious Agents and Cancer. 2021;16:41.

17. Balamurugan R, Rajendiran E, George S, Samuel GV, Ramakrishna BS. Real-time polymerase chain reaction quantification of specific butyrateproducing bacteria, Desulfovibrio and enterococcus faecalis in the feces of patients with colorectal cancer. J Gastroenterol Hepatol. 2008;23(8 Pt 1):1298-303.

18. Michael DW, Tiffany LS, Wendy AJ, Stephen RG, Mark MH. Prospective case-cohort study of intestinal colonization with enterococci that produce extracellular superoxide and the risk for colorectal adenomas or cancer. Am J Gastroenterol. 1998;93(12):2491-500.

19. Boleij A, van Gelder MMHJ, Swinkels DW, Tjalsma H. Clinical importance of streptococcus gallolyticus infection among colorectal Cancer patients: systematic review and Meta-analysis. Clin Infect Dis. 2011;53(9):870-8.

20. Mahmoudvand S, Zamani K, Safaei A, Khashei R, Motamedifar M, Azizi $Z$, et al. No detection of streptococcus gallolyticus and helicobacter pylori in colorectal Cancer tissue samples in shiraz. Iran Iran J Cancer Prev. 2017;10(1):e6337.

21. Abdulamir AS, Hafidh RR, Bakar FA. Molecular detection, quantification, and isolation of streptococcus gallolyticus bacteria colonizing colorectal tumors: inflammation-driven potential of carcinogenesis via IL-1, COX-2, and IL-8. Mol Cancer. 2010;9:249-9.

22. Farajzadeh Sheikh A, Masjedi Zadeh AR, Saki M, Khani P, Hashemi SJ, Shahin Zadeh S, et al. Detection of streptococcus gallolyticus in colorectal cancer and inflammatory bowel disease patients compared to control group in southwest of Iran. Mol Biol Rep. 2020;47(11):8361-5.

23. Hale VL, Jeraldo P, Chen J, Mundy M, Yao J, Priya S, et al. Distinct microbes, metabolites, and ecologies define the microbiome in deficient and proficient mismatch repair colorectal cancers. Genome Medicine. 2018;10(1):78

24. Kashani N, Abadi AB, Rahimi F, Forootan M. FadA-positive Fusobacterium nucleatum is prevalent in biopsy specimens of Iranian patients with colorectal cancer. New Microbes New Infect. 2020;34:100651.

25. Kovacs Z, Jung I, Csernak E, Szentirmay Z, Banias L, Rigmanyi G, et al. DNA extraction from paraffin embedded colorectal carcinoma samples: a comparison study of manual vs automated methods, using four commercially kits. World journal of clinical oncology. 2019;10(9):307-17.

\section{Publisher's Note}

Springer Nature remains neutral with regard to jurisdictional claims in published maps and institutional affiliations.

Ready to submit your research? Choose BMC and benefit from:

- fast, convenient online submission

- thorough peer review by experienced researchers in your field

- rapid publication on acceptance

- support for research data, including large and complex data types

- gold Open Access which fosters wider collaboration and increased citations

- maximum visibility for your research: over $100 \mathrm{M}$ website views per year

At BMC, research is always in progress.

Learn more biomedcentral.com/submissions 